\title{
A comparison of dairy cattle breeding designs that use genomic selection
}

\author{
M. Lillehammer, ${ }^{\star 1}$ T. H. E. Meuwissen, $†$ and A. K. Sonesson* \\ *Nofima Marin, PO Box 5010, 1432 Ås, Norway \\ †University of Life Sciences, Department of Animal and Aquacultural Sciences, PO Box 5003, 1432 Ås, Norway
}

\begin{abstract}
Different dairy cattle breeding schemes were compared using stochastic simulations, in which the accuracy of the genomic breeding values was dependent on the structure of the breeding scheme, through the availability of new genotyped animals with phenotypic information. Most studies that predict the gain by implementing genomic selection apply a deterministic approach that requires assumptions about the accuracy of the genomic breeding values. The achieved genetic gain, when genomic selection was the only selection method to directly identify elite sires for widespread use and progeny testing was omitted, was compared with using genomic selection for preselection of young bulls for progeny testing and to a conventional progeny test scheme. The rate of inbreeding could be reduced by selecting more sires every year. Selecting 20 sires directly on their genomic breeding values gave a higher genetic gain than any progeny testing scheme, with the same rate of inbreeding as the schemes that used genomic selection for preselection of bulls before progeny testing. The genomic selection breeding schemes could reduce the rate of inbreeding and still increase genetic gain, compared with the conventional breeding scheme. Since progeny testing is expensive, the breeding scheme omitting the progeny test will be the cheapest one. Keeping the progeny test and use of genomic selection for preselection still has some advantages. It gives higher accuracy of breeding values and does not require a complete restructuring of the breeding program. Comparing at the same rate of inbreeding, using genomic selection for elite sire selection only gives a $13 \%$ increase in genetic gain, compared with using genomic selection for preselection. One way to reduce the costs of the scheme where genomic selection was used for preselection is to reduce the number of progeny tested bulls. This was here achieved without getting lower genetic gain or a higher rate of inbreeding.
\end{abstract}

Received June 9, 2010.

Accepted September 22, 2010.

${ }^{1}$ Corresponding author: marie.lillehammer@nofima.no
Key words: genomic selection, breeding design, progeny test

\section{INTRODUCTION}

In genomic selection, breeding values are estimated based on thousands of molecular markers, instead of own performance and family information. Using molecular markers, accurate breeding values can be obtained for animals of both sexes early in life, which can be used to shorten the generation interval for dairy cattle by omitting the progeny test (Schaeffer, 2006). Compared with conventional BLUP breeding values (Henderson, 1984), genomic selection provides more accurate breeding values for young animals, but the conventional BLUP breeding values are more accurate when the animals get older and get their progeny test records. This availability of early genomic breeding values provides an opportunity to choose between selection candidates based on accurate genomic breeding values early in life, and selection based on more accurate progeny-based BLUP breeding values later in life. Some farmers may prefer to use older bulls with daughter proofs, which would increase the generation interval and decrease the benefit of using genomic selection (König et al., 2009). Genomic selection could contribute to higher genetic gain without increased inbreeding, because of increased accuracy of early breeding values, which makes it possible to increase genetic gain without increasing selection intensity (Daetwyler et al., 2007). Schaeffer (2006) showed that genomic selection has the potential to reduce the generation interval of dairy cows and bulls substantially, causing increased genetic gain and reduced costs of the breeding program, assuming an accuracy of the genomic breeding values of 0.75 . These results were obtained by selecting elite sires based on genomic breeding values at a young age, and the main contribution to the increased genetic gain was the shortened generation interval.

In several studies that use deterministic approaches, accuracy is assumed to be fixed and the schemes are compared under that assumption (e.g., Schaeffer, 2006; König et al., 2009). However, it has been shown that without re-estimation of marker effects, the accuracy 
of genomic breeding values for a trait under selection decreases rapidly (Muir, 2007; Sonesson and Meuwissen, 2009). By using stochastic simulation, it is possible to study the effect of different breeding schemes on genetic gain without treating accuracy as a fixed value, but rather as an outcome of the tested scheme, and to study how accuracy changes over time under schemes with different possibilities for re-estimation of marker effects. The aim of this study was to compare dairy cattle breeding schemes when accuracies of genomic breeding values are not given but are dependent on the re-estimation of marker effects. The population structure, including the selection intensity and the number of selected sires, was chosen to reflect the Norwegian dairy cattle population. A conventional breeding scheme in which no genomic selection was used was compared with a breeding scheme in which genomic selection was used to preselect young bulls for progeny testing and to a breeding scheme in which genomic selection was used to select elite sires directly at a younger age.

\section{MATERIALS AND METHODS}

All breeding schemes were started from a base population, which was simulated according to the FisherWright population model (Fisher, 1930; Wright, 1931). This historical population had an effective population size of 200 and was simulated for 2,000 generations using random mating to create mutation-drift balance. The genome was simulated to consist of 30 pairs of 100$\mathrm{cM}$ chromosomes. Polymorphisms and recombinations were simulated as in Sonesson and Meuwissen (2009). Among the SNP created, 100 from each chromosome were randomly selected as QTL. The QTL effects were sampled from a gamma distribution with a shape parameter of 0.4 and a scale parameter of 1.66 (Hayes and Goddard, 2001). From each chromosome, 500 SNP were sampled to be markers to be used for estimating genomic breeding values. The animals from the last generation of this simulation were randomly mated to create a base generation of 3,000 animals (generation $0)$. For the schemes involving genomic selection, the 3,000 animals in generation 0 were assumed genotyped and progeny tested and used to estimate SNP effects. This reference population was updated yearly, with new genotyped and progeny tested sires generated by the selection scheme. This should reflect a typical dairy cattle structure where some historically progeny-tested bulls are genotyped and used to estimate SNP effects and younger bulls are added when they get progeny test records.

True breeding values (TBV) were calculated for all individuals as a sum of the QTL effects:

$$
T B V_{i}=\sum_{j=1}^{3000} x_{i j}\left(g_{j 1}-g_{j 2}\right)
$$

(Sonesson and Meuwissen, 2009), where $x_{i j}$ is the number of copies that individual $i$ has at the $j$ th QTL position of the first QTL allele, and $g_{j k}$ is the effect of the $k$ th QTL allele at the $j$ th position. The simulated trait was given a heritability of 0.15 , which reflects the heritability of the total merit index for milk production (Sonesson et al., 2008). In addition, heritability was varied between 0.01 and 0.30 to study the effect of heritability on the results. Females received a phenotypic record before $3 \mathrm{yr}$ of age, such that female selection candidates aged 3 and above were assumed to have own performance information. Phenotypes were simulated by adding a normally distributed random error term to the true breeding value.

Marker effects were estimated using the BLUP method (Meuwissen et al., 2001). The statistical model used to estimate individual marker effects was

$$
y_{i}=\mu+\sum_{j=1}^{n} X_{i j} a_{j}+e_{i},
$$

where $y_{i}$ is the record of individual $i ; \mu$ is the overall mean; $X_{i j}$ is the marker genotype; $a_{j}$ is the random effect of the $j$ th marker, with variance equal to the total genetic variance divided by the number of markers; and $e_{i}$ is a random residual. Re-estimation of SNP effects was performed yearly for the schemes involving genomic selection, by adding the newly genotyped bulls to the reference population after they get their progeny records.

To make stochastic simulation computationally possible simulated population sizes were smaller than those of the real Norwegian Red dairy cattle population. However, the number of selected males and the selected fractions of males and females for milk production traits were equal to that of Norwegian Red dairy cattle. Hence, selection intensities are expected to be similar to those of the real-life population. An overview of the rescaled breeding scheme is given in Figure 1. Selection of sires was performed in 2 steps. First, 125 young bulls were preselected for progeny testing based on EBV. Then, 12 elite sires were selected based on the results from the progeny test. Every tested bull was evaluated based on a simulated progeny test record, with accuracy as expected from 260 daughters with a single record for the simulated trait. This reflects the average size for the progeny groups in Norway. The elite sires were mated to the 1,500 females with the 


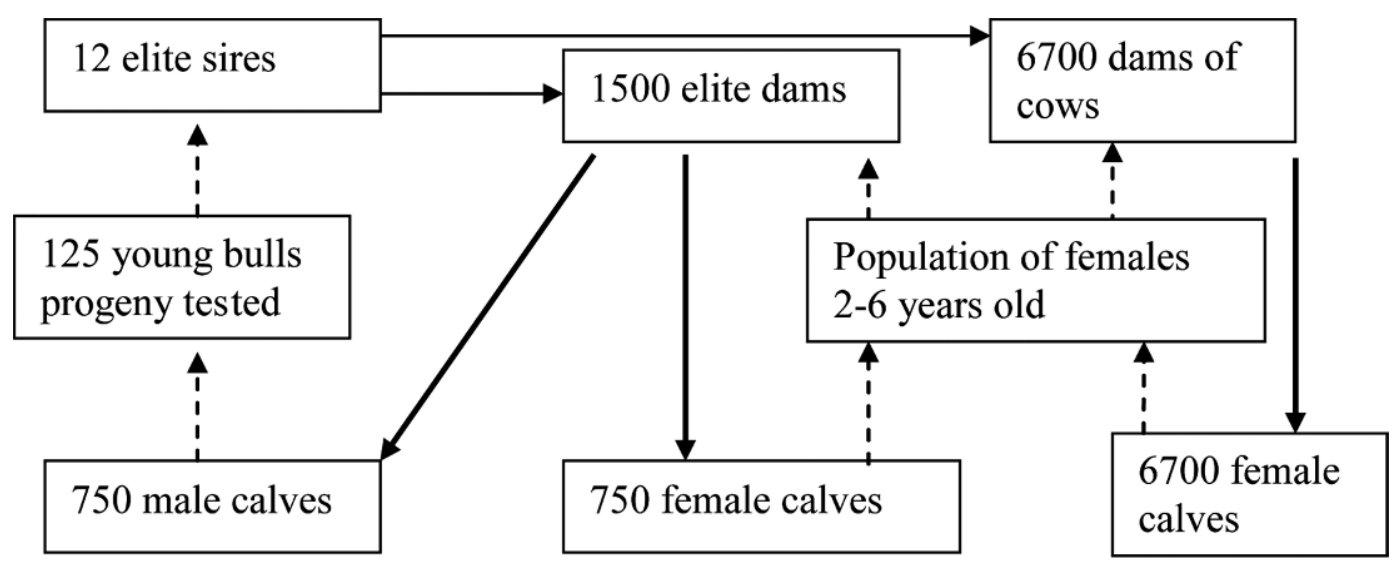

Figure 1. Overview of the rescaled conventional breeding scheme. Solid arrows represent mating (thin line) or progeny generation (thick line). Dotted arrows represent that animals move from one category to another due to aging or selection. Males were selected in a 2-step procedure, and unselected males were culled after each selection, whereas females remained in the population until culling and were selection candidates as long as they were alive. Females were randomly culled between the age of 3 and 6 yr. The selected elite dams were always the females with the highest EBV, whereas dams of cows were selected randomly among the $90 \%$ of the females with the highest breeding values.

highest breeding values to produce 750 new male selection candidates and 750 female selection candidates. In addition, the elite sires were mated to 6,700 females chosen randomly from the top $90 \%$ of the female population, based on breeding values, to produce commercial replacement heifers for the cow population. Females became selection candidates at the age of $2 \mathrm{yr}$ and remained candidates until they passed $6 \mathrm{yr}$ of age. This pool of females served as selection candidates both for the selection of dams of bulls and for dams of new cows. One-third of the females were culled at random every year from 3 yr of age.

For the conventional breeding scheme (CONV), the selection steps were performed based on conventional BLUP breeding values. Two different strategies for implementing genomic selection were tested. For both the strategies, the male selection candidates were genotyped and genomic breeding values (GEBV) estimated by summing the marker effects:

$$
G E B V_{i}=\sum_{j=1}^{n} x_{i j} a_{j}
$$

where $a_{j}$ is the BLUP estimate of the $j$ th SNP effect. The accuracy of the genomic breeding values was calculated as the correlation between the estimated genomic breeding values and the true breeding values (Sonesson and Meuwissen, 2009). The genomic breeding values were either used to pre-select young bulls for progeny testing (PS) or used to select elite sires directly (GS), omitting the progeny test. Applying GS could thereby shorten the male generation interval from 6 to $3 \mathrm{yr}$ and increase the number of females born in the breeding population, because omitting the progeny test would cause all female calves born to be selection candidates

Progeny-tested bulls (PS and CONV) or elite bulls (GS) received a progeny test record at the age of 5 to $6 \mathrm{yr}$. The progeny test records were calculated as daughter yield deviations (DYD) directly, using the formula

$$
D Y D_{i}=\frac{1}{2} T B V_{i}+\sqrt{\frac{\frac{3}{4} \sigma_{g}^{2}+\sigma_{e}^{2}}{N_{i}}} \times r_{i},
$$

where $N_{i}$ is the number of daughters of sire $i$ and $r_{i}$ is a random standard normal deviate. Hence, individual test-daughters were not simulated, and the progeny test record was assumed to be based on one record per daughter. The DYD were used to obtain more accurate BLUP breeding values for elite sire selection in the schemes PS and CONV. In the PS and GS schemes, sires were added to the reference population after they got their progeny test record, to re-estimate the marker effects. The genetic variance $\left(\sigma_{g}^{2}\right)$ was standardized to 30 in all situations, and the error variance $\left(\sigma_{e}^{2}\right)$ was adjusted to create phenotypic records with the desired heritability (Sonesson and Meuwissen, 2009).

In addition to simply replacing the current selection method by genomic selection, the effect of varying the number of selected bulls within each scheme was explored. In the PS schemes, the effect of varying the number of progeny-tested bulls between 60 and 125 was studied. The number of elite sires was kept constant at 12 for all the PS schemes. When the number 
of bulls to be progeny tested was decreased, each test bull could have more progeny (PS_A schemes) or fewer cows could be used for progeny testing, increasing the number of cows mated to elite sires (PS_B schemes). For a trait with heritability 0.15 , both alternatives were tested for 60 and 80 progeny tested bulls (PS_60_260, PS_60_540, PS_80_260, PS_80_406), and 100 bulls were tested with an increased number of progeny per test bull (PS_100_325), where the numbers stated after PS denotes the number of tested bulls and the number of daughters per bull, respectively. For other heritabilities, testing 125 bulls (PS_125_260) was compared with testing 60 bulls where each tested bull gets more progeny (PS_60_540). In the GS schemes, the overall selection fraction was varied, by varying the number of selected sires. The schemes were denoted GS_xx, where $\mathrm{xx}$ is the number of selected sires each year. The number of elite sires was varied from 12 to 40. For a trait with heritability of $0.15 ; 12,20,30$ and 40 elite sires were tested, whereas for other heritabilities, only 12 and 40 elite sires were tested.

For each scheme, 100 replicates were run, and all schemes were run for 19 yr. Genetic gain and rate of inbreeding were reported as an average for yr 13 to 19 of the simulation, which corresponds to 1 generation for the CONV and PS schemes and 2 generations for the GS schemes. Omitting the first years of the simulation when results are reported avoids the problem of the population structure at the start of the scheme, where all animals are of the same age, affecting the results. Accuracy of the genomic breeding values was reported at yr 19 of the simulation in the tables, and the change in accuracy over time is presented in Figure 3.

\section{RESULTS}

The CONV scheme gave a rate of inbreeding of 0.0025 per year and genetic gain of $0.22 \sigma_{\mathrm{g}}$ (genetic SD) per year. The PS_125_260 scheme caused an increase in genetic gain of $13 \%$, with a $33 \%$ decrease in the rate of inbreeding compared with CONV (Table 1). The GS_12 scheme increased the genetic gain by $33 \%$ and decreased the rate of inbreeding by $2 \%$ compared with CONV. Using PS, changing the number of preselected bulls had only minor effects on the results, the increase in genetic gain varied between 11 and $14 \%$, whereas the decrease in rates of inbreeding varied between 30 and $37 \%$. The accuracy of the genomic breeding values was slightly lower when fewer bulls were progeny tested, but as the final elite-sire selection was done by progeny testing, this had small effects on the genetic gain. Using GS, the accuracy of the genomic breeding values increased as the number of selected sires increased. The increase in accuracy did not compensate for the reduced
Table 1. Rate of inbreeding $(\Delta \mathrm{F})$, genetic gain $(\Delta \mathrm{G})$, and accuracy of the genomic breeding values when $h^{2}=0.15$

\begin{tabular}{lccc}
\hline Breeding scheme & \multicolumn{1}{c}{$\Delta \mathrm{F}^{2}$} & $\Delta \mathrm{G}^{2}$ & Accuracy $^{3}$ \\
\hline CONV & 1.00 & 1.00 & -4 \\
PS_125_260 & 0.67 & 1.13 & 0.70 \\
PS_100_325 & 0.66 & 1.13 & 0.70 \\
PS_80_406 & 0.69 & 1.14 & 0.69 \\
PS_80_260 & 0.70 & 1.13 & 0.68 \\
PS_60_542 & 0.68 & 1.13 & 0.68 \\
PS_60_260 & 0.63 & 1.11 & 0.66 \\
GS_12 & 0.98 & 1.33 & 0.61 \\
GS_20 & 0.70 & 1.28 & 0.61 \\
GS_30 & 0.47 & 1.25 & 0.63 \\
GS_40 & 0.36 & 1.20 & 0.63 \\
\hline
\end{tabular}

${ }^{1} \mathrm{CONV}$ denotes a conventional breeding scheme; PS_xx_yy denote breeding schemes using genomic breeding values for preselection of young bulls, where $\mathrm{xx}$ is the number of progeny tested bulls and yy is the size of the progeny group; GS_xx are schemes where genomic selection is used to select elite sires directly, where $\mathrm{xx}$ denotes the number of selected elite sires.

${ }^{2}$ Average of years 13 to 19 , relative to the conventional scheme $(\Delta \mathrm{F}=$ $0.0025, \Delta \mathrm{G}=0.22 \sigma_{\mathrm{g}}$ ).

${ }^{3}$ Accuracy of the genomic breeding values in yr 19 of the simulations. ${ }^{4}$ No accuracy is given, because no genomic breeding values are calculated for CONV.

selection intensity when the number of selected sires increased. When the number of sires was increased, both genetic gain and rate of inbreeding decreased. It was possible to obtain an increase in genetic gain together with a decrease in the rate of inbreeding, relative to the conventional scheme.

When heritability was increased to 0.3 , genetic gain of CONV was increased to $0.23 \sigma_{\mathrm{g}} / \mathrm{yr}$ and rate of inbreeding was reduced to $0.0024 / \mathrm{yr}$. When heritability was reduced, CONV gave reduced genetic gain $(0.20$ $\sigma_{\mathrm{g}} / \mathrm{yr}$ when $h^{2}=0.05$ and $0.16 \sigma_{\mathrm{g}} / \mathrm{yr}$ when $\left.h^{2}=0.01\right)$ and increased inbreeding $\left(0.0031 / \mathrm{yr}\right.$ when $h^{2}=0.05$ and $0.0047 / \mathrm{yr}$ when $\left.h^{2}=0.01\right)$. Table 2 summarizes how PS and GS performed relative to CONV. The results when genomic selection was applied were less affected by heritability than the CONV results, causing genomic selection to be more favorable for lowly heritable traits. The PS scheme showed a higher accuracy for the genomic breeding values than the GS scheme, except when heritability was 0.01 . The PS schemes had more genotyped bulls with progeny; that is, more DYD for re-estimation of SNP effects are added every year. In addition, the generation interval was long, which results in a relatively small annual change in SNP effects. When heritability was very low, the number of progeny per bull became more important, favoring GS and the PS_60_540 scheme, in which the number of progeny per bull was higher.

For all heritabilities $>0.01$, accuracy was increased when the number of selected sires within the GS scheme was increased, but it could not compensate for the re- 


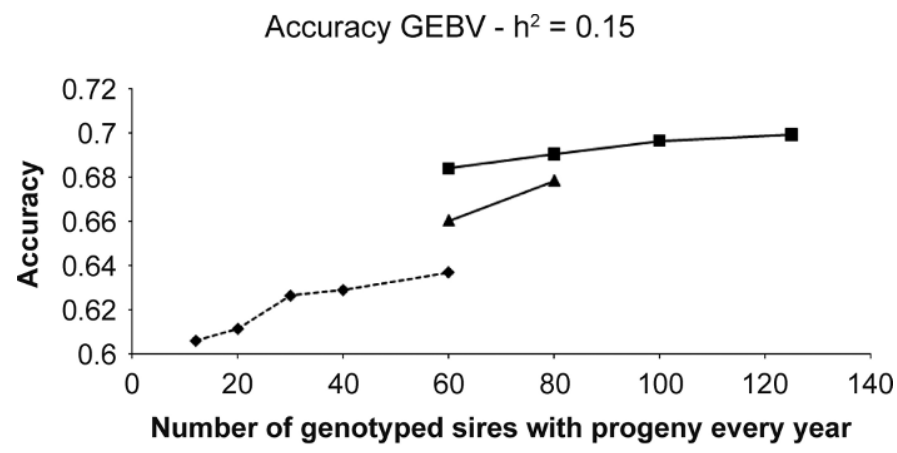

$\cdots \rightarrow$ GS $\rightarrow$ PS_A $\leftarrow$ PS_B

Figure 2. Accuracy of the genomic breeding values (GEBV) after $19 \mathrm{yr}$ for a trait with heritability $=0.15$. In GS, genomic breeding values were used to select elite sires directly. In PS_A and PS_B, genomic breeding values were used to preselect young bulls. In PS_A, the size of the progeny group was increased when the number of test bulls decreased, whereas in PS_B, the size of the progeny group was kept constant.

duced selection intensity, so genetic gain increased with a lower number of selected sires. However, the reduction in rate of inbreeding was higher than the reduction in genetic gain when the number of sires was increased, and increasing the number of sires could be needed to decrease the rate of inbreeding. To get the same low rate of inbreeding with GS that was obtained with PS, 20 or more elite sires had to be selected every year (Table 1). The benefit of using GS_20 compared with any of the PS schemes was a $13 \%$ increase in genetic gain (Table 1).

Figure 2 shows that the accuracy of the genomic breeding values increased with increasing number of genotyped sires with offspring, but that the difference in accuracy between the schemes was not fully explained by different number of sires. All categories of schemes were tested with 60 sires with progeny, showing that differences in accuracy of the genomic breeding values remained when the number of sires with progeny records was kept constant. Figure 3 further shows that the schemes differ in how the accuracies change over time. The differences between the schemes increased over time, as schemes with many progeny tested sires got increased accuracy, whereas accuracy decreased for schemes with few sires with progeny. The changes in accuracy every year were quite small (Figure 3) but accumulated over time to create a considerable difference between the schemes (Figure 2).

\section{DISCUSSION}

The optimal breeding scheme depends on how the different result parameters are weighted against each other. The GS schemes gave the highest genetic gain, even when schemes were compared at the same rate of inbreeding. The tested GS schemes were also cheaper than both PS and CONV because progeny testing is more expensive than genotyping, assuming the number of genotyped bull calves to be kept constant at 750 . The PS schemes gave a higher accuracy of the genomic breeding values than the GS schemes (Tables 1 and 2 ). The advantage of reducing the generation interval is therefore lower than what has been reported from deterministic studies, when the accuracy of genomic breeding values has been regarded as unaffected by the breeding scheme (Schaeffer, 2006; König et al., 2009).

Implementing genomic selection caused a decrease in rate of inbreeding both when used to select elite sires (GS) and when used to preselect young bulls for progeny testing (PS). All schemes were run without attempting to restrict inbreeding, and this caused a high rate of inbreeding in the conventional scheme, because half sibs have similar conventional breeding values and have a high risk of being co-selected in the preselection step. When genomic selection is used, close relatives will have less similar breeding values, causing a reduction in the

Table 2. Rate of inbreeding $(\Delta \mathrm{F})$, genetic gain $(\Delta \mathrm{G})$, and accuracy (Acc.) of the genomic breeding values when heritability is varied

\begin{tabular}{|c|c|c|c|c|c|c|c|c|c|}
\hline Breeding scheme ${ }^{1}$ & \multicolumn{3}{|c|}{$h^{2}=0.01$} & \multicolumn{3}{|c|}{$h^{2}=0.05$} & \multicolumn{3}{|c|}{$h^{2}=0.30$} \\
\hline CONV & 1.0 & 1.0 & $-^{4}$ & 1.0 & 1.0 & - & 1.0 & 1.0 & - \\
\hline PS_125_260 & 0.76 & 1.20 & 0.51 & 0.69 & 1.15 & 0.64 & 0.57 & 1.11 & 0.72 \\
\hline PS_60_542 & 0.66 & 1.26 & 0.54 & 0.62 & 1.14 & 0.64 & 0.67 & 1.09 & 0.69 \\
\hline GS_12 & 0.64 & 1.70 & 0.60 & 0.93 & 1.40 & 0.61 & 1.14 & 1.29 & 0.60 \\
\hline GS_40 & 0.27 & 1.44 & 0.57 & 0.33 & 1.24 & 0.62 & 0.35 & 1.17 & 0.63 \\
\hline
\end{tabular}

${ }^{1}$ CONV denotes a conventional breeding scheme; PS_xx_yy are schemes where genomic selection is used to preselect xx bulls that are progeny tested, and where yy denotes the size of the progeny group; GS_12 and GS_40 are schemes where genomic breeding values were used to select elite sires directly, selecting 12 or 40 elite sires, respectively.

${ }^{2}$ Average of years 13 to 19 , relative to the conventional scheme.

${ }^{3}$ Accuracy of the genomic breeding values in yr 19 of the simulations.

${ }^{4} \mathrm{No}$ accuracy is given, because no genomic breeding values are calculated for CONV. 


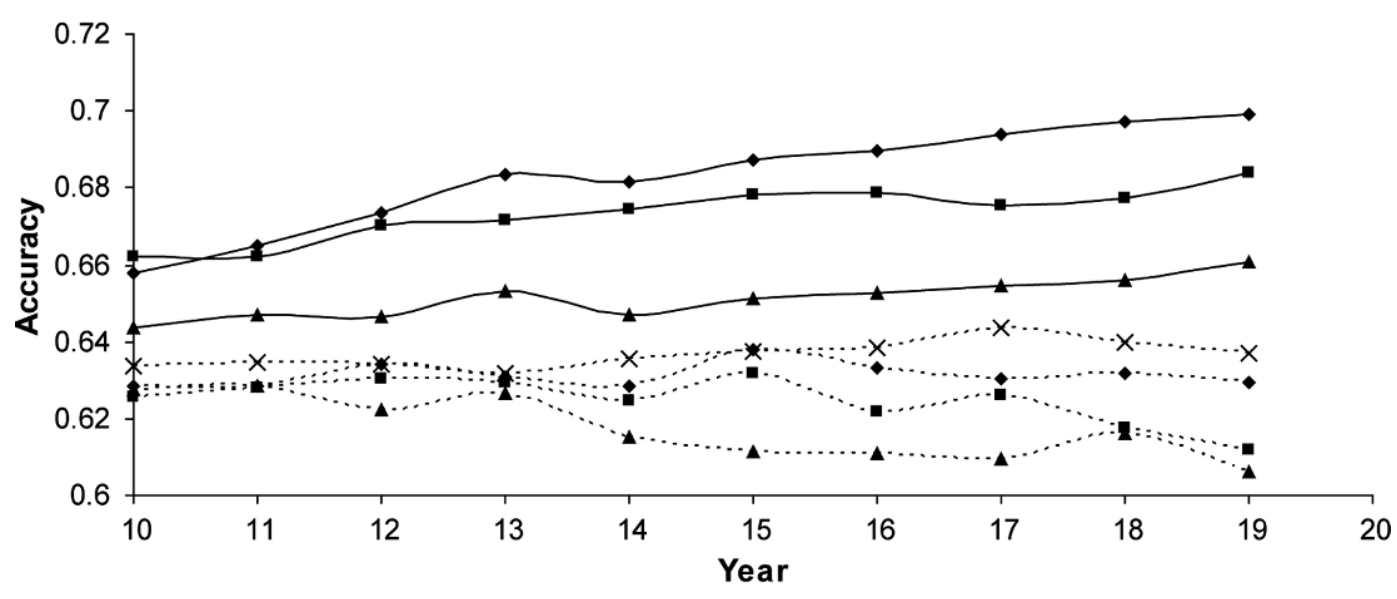

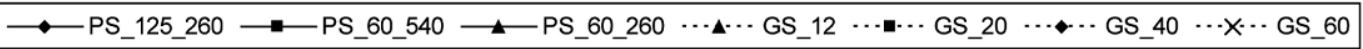

Figure 3. Development of accuracy of the genomic breeding values (GEBV) of 7 breeding schemes from 10 to 19 yr after the scheme was started. PS_xx_yy are schemes in which genomic selection is used to preselect xx bulls that are progeny tested and where yy denotes the size of the progeny group. The GS_xx are schemes in which genomic breeding values were used to select elite sires directly at a younger age, where $\mathrm{xx}$ denotes the number of selected elite sires.

rate of inbreeding (Daetwyler et al., 2007). The results from the PS schemes quantify this reduction to be more than $30 \%$. In the GS schemes, the reduced generation interval compensated for the reduction in inbreeding caused by the genomic breeding values, and the rate of inbreeding was thereby similar to that achieved by the conventional scheme. To achieve a reduction in the rate of inbreeding with the GS scheme, the number of elite sires needed to be increased. Then, the genetic gain was also reduced, but remained higher than with any of the schemes involving progeny testing (Table 1).

The heritability of the total merit index of Norwegian Red Cattle was low relative to that in other breeds because of the high weight of functional traits such as clinical mastitis, which has a low heritability. As shown in Table 2, the heritability of the trait affected the accuracy of the genomic breeding values only when it became very low, but the relative superiority of genomic selection schemes was reduced as the heritability of total merit index increased.

Among the parameters varied in this study, the number of genotyped sires with progeny was shown to explain most, but not all, of the variation in accuracy of the breeding values (Figure 2). Other factors affecting the accuracy are the size of the progeny group and the generation interval. The PS scheme gives a higher accuracy than the GS at the same number of genotyped sires with progeny (Figure 2). In the PS schemes, estimates of SNP effects involve training records of the sires of the young bulls, whereas in the GS schemes it involves grandsires and older relatives. Hence, the estimates of SNP effects are based on information that is only 1 generation old in the PS schemes, whereas it is 2 generations old in the GS schemes. The shortened generation interval of GS increases the annual change in the SNP effects, and thereby increases the need for re-estimated SNP effects. This causes a reduction in accuracy in the GS schemes, because the genetic ties between the reference population and the test population have a strong influence on the accuracy of genomic breeding values (Habier et al., 2007; Lund et al., 2009). In this study, it was assumed that the breeding population was the only source of newly genotyped animals to be added to the reference population for re-estimation of SNP effects. Cooperation between breeding populations can increase the size of the reference population and decrease the need for new genotyped animals with progeny from each population, assuming that the genetic ties between the populations are strong enough that this common reference population is relevant for all participating populations.

Accuracy also differed between the PS schemes with different sizes of progeny groups (Figure 2). The reduction in accuracy when reducing the number of progeny-tested sires can be compensated for by increasing the size of the progeny group. However, it might be economically beneficial to keep the size of the progeny groups constant and mate more cows to elite sires to increase the overall productivity of the population. For all the tested schemes, 260 progeny was sufficient to give good accuracy when heritability was 0.05 or higher, but the number of progeny per sire becomes a limiting 
factor with a heritability of 0.01 . This result has also been found in studies comparing different progeny testing schemes, where the optimal size of the daughter group is higher for lowly heritable traits (Willam et al., 2002).

In this study, the number of QTL was kept constant at 100 per chromosome, and the QTL effects were sampled from a gamma distribution with a shape parameter of 0.4 and a scale parameter of 1.66 . The accuracy of the genomic breeding values might be dependent on the true genetic architecture, which is unknown. However, the BLUP method used to estimate the SNP effects (Meuwissen et al., 2001) has been shown to be robust against differences in the number of QTL and the distribution of QTL effects (Daetwyler et al., 2008; Meuwissen and Goddard, 2010).

The accuracy of breeding values is important not only through its effect on genetic gain, but also for marketing purposes, because a lower accuracy of the breeding values increases the risk of poor performance of single animals and larger variation between the selected animals. This is of limited importance when the breeding values are used in the PS schemes, because the variability of selected young bulls will be reduced after progeny testing, but it is highly relevant for the GS schemes. König et al. (2009) addressed this by assuming that a fraction of the farmers would systematically choose older bulls, which already have other offspring with records, to ensure the high quality of bulls used for insemination. This would increase the generation interval and hence decrease genetic gain. Using genomic selection for preselection of young bulls is also a lowrisk approach, as it requires only minor changes to the current breeding program.

Generally, the schemes rank similarly, independent of heritability of the trait under selection. The main difference is that genomic selection becomes more favorable when heritability is reduced, because the accuracy of genomic breeding values is less affected by heritability than by the accuracy of conventional BLUP breeding values. The relative accuracy of genomic breeding values at low heritability is caused by the increased use of information from relatives in genomic breeding values, which is made possible by the marker data, providing detailed identity-by-descent relationships at the DNA level. Hence, as heritability decreases, phenotypic information becomes less relevant and the use of information from relatives becomes increasingly important. Selection is usually performed for several traits with different heritabilities, whereas the total merit index of dairy cattle has a heritability of about 0.15 (Sonesson et al., 2008). The results reported for $h^{2}=0.15$ (Table 1) can therefore be seen as an indication of how the total genetic gain and inbreeding would be affected by the implementation of genomic selection. The difference between results obtained for the different heritabilities (Table 2) shows how traits of different heritabilities can change their contribution to the overall genetic gain if genomic selection is implemented. Because genomic selection increases genetic gain most in lowly heritable traits, their effect on the total genetic gain would increase with the implementation of genomic selection. This should be seen as a positive effect of implementing genomic selection, because lowly heritable traits are usually hard to improve through selection. In the current breeding goals for dairy cattle, several functional traits such as disease resistance and reproduction have low heritabilities. These traits have received more focus in recent years, both due to economical importance and due to their effect on animal welfare (AndersenRanberg et al., 2005; Miglior et al., 2005). Genomic selection increases the contribution from these traits to the overall genetic gain and hence results in a more sustainable breeding scheme.

\section{CONCLUSIONS}

The breeding scheme giving highest genetic gain would be GS selecting 12 elite sires every year (GS_12), and this scheme will give the same rate of inbreeding as CONV. Selecting more sires can cause a decrease in genetic gain together with a decrease in rate of inbreeding. Selecting 20 sires within GS would give a higher genetic gain than any progeny testing scheme, with the same rate of inbreeding as the PS schemes. However, GS_20 gives only 13\% increased genetic gain compared with the PS schemes. The PS schemes give a higher accuracy of the breeding values of the elite sires, because of progeny testing, and are more similar to the current breeding scheme, so implementing a PS scheme would be a less dramatic change in the breeding program. The number of sires that are progeny tested could be reduced without losing genetic gain or increasing the rate of inbreeding. When fewer sires are progeny tested, fewer cows are needed for the progeny testing, and an additional benefit for the farmers would be that more cows could be mated to elite sires, if the size of the progeny group is not increased.

\section{ACKNOWLEDGMENTS}

The authors thank GENO breeding and AI organization (Ås, Norway) for valuable input about the current breeding scheme, selection fractions and population structure. This project was funded from the Norwegian Research Council, project no. 186862. Calculations were done on the TITAN computer cluster at University of Oslo, Norway. 


\section{REFERENCES}

Andersen-Ranberg, I. M., G. Klemetsdal, B. Heringstad, and T. Steine. 2005. Heritabilities, genetic correlations, and genetic change for female fertility and protein yield in Norwegian dairy cattle. J. Dairy Sci. 88:348-355.

Daetwyler, H. D., B. Villanueva, P. Bijma, and J. A. Woolliams. 2007. Inbreeding in genome-wide selection. J. Anim. Breed. Genet. 124:369-376.

Daetwyler, H. D., B. Villanueva, and J. A. Woolliams. 2008. Accuracy of predicting the genetic risk of disease using a genome-wide approach. PLoS ONE 3:e3395.

Fisher, R. A. 1930. The Genetical Theory of Natural Selection. Clarendon Press, Oxford, UK.

Habier, D., R. L. Fernando, and J. C. M. Dekkers. 2007. The impact of genetic relationship information on genome-assisted breeding values. Genetics 177:2389-2397.

Hayes, B. J., and M. E. Goddard. 2001. The distribution of the effects of genes affecting quantitative traits in livestock. Genet. Sel. Evol. 33:209-229.

Henderson, C. 1984. Applications of Linear Models in Animal Breeding. Guelph University Press, Guelph, Canada.

König, S., H. Simianer, and A. Willam. 2009. Economic evaluation of genomic breeding programs. J. Dairy Sci. 92:382-391.

Lund, M. S., G. Su, U. S. Nielsen, and G. P. Aamand. 2009. Relation between accuracies of genomic predictions and ancestral links to the training data. Pages 162-166 in Proceedings of the 2009 Interbull meeting, Barcelona, Spain. Interbull, Uppsala, Sweden.
Meuwissen, T. H. E., and M. E. Goddard. 2010. Accurate prediction of genetic values for complex traits by whole-genome resequencing. Genetics 185:623-631.

Meuwissen, T. H. E., B. J. Hayes, and M. E. Goddard. 2001. Prediction of total genetic value using genome-wide dense marker maps. Genetics 157:1819-1829.

Miglior, F., B. L. Muir, and B. J. Van Doormaal. 2005. Selection indices in Holstein cattle of vaious countries. J. Dairy Sci. 88:12551263.

Muir, W. M. 2007. Comparison of genomic and traditional BLUPestimated breeding value accuracy and selection response under alternative trait and genomic parameters. J. Anim. Breed. Genet. 124:342-355.

Schaeffer, L. R. 2006. Strategy for applying genome-wide selection in dairy cattle. J. Anim. Breed. Genet. 123:218-223.

Sonesson, A., T. Meuwissen, and A. Cromie. 2008. Genomic selection in Irish dairy cattle breeding scheme. Irish Cattle Breeding Federation. http://www.icbf.com/publications/files/Genomic_Selection_in_Irish_dairy_cattle_breeding_Report_Dec_2008.pdf

Sonesson, A. K. and T. H. E. Meuwissen. 2009. Testing strategies for genomic selection in aquaculture breeding programs. Genet. Sel. Evol. 41:37.

Willam, A., C. Egger-Danner, J. Sölkner, and E. Gierzinger. 2002. Optimization of progeny testing schemes when functional traits play an important role in the total merit index. Livest. Prod. Sci. $77: 217-225$

Wright, S. 1931. Evolution in Mendelian populations. Genetics 16:97159 . 